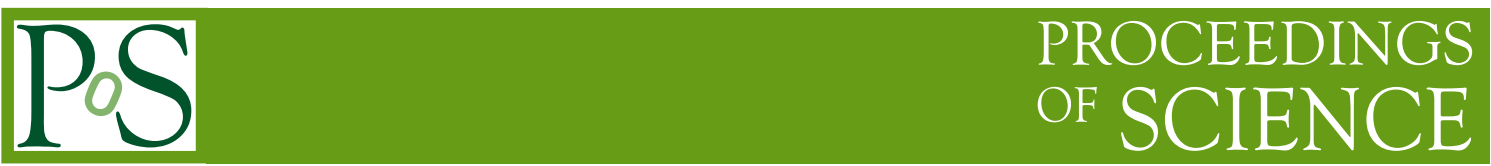

\title{
Two Early Exotic searches with dijet events at ATLAS
}

\author{
Reyhaneh Rezvani ${ }^{* \dagger}$ \\ University of Toronto, Department of Physics \\ E-mail: rrezvani@physics.utoronto.ca
}

\begin{abstract}
This document summarises two exotic searches in dijet events. In the first search, narrow resonances of the dijet invariant mass from the decay of new heavy particles have been investigated. An integrated luminosity of $3.1 \mathrm{pb}^{-1}$ from $7 \mathrm{TeV}$ collision data in LHC has been used. No significant evidence of new particles is found, and upper limits have been set on the product of the cross-section and detector acceptance. The theoretical model considered is the composite excited quark production, decaying to dijets. A 95\% confidence-level mass exclusion region of the composite excited quark $\mathrm{q}^{*}$ has been determined $\left(0.50<m_{q^{*}}<1.53 \mathrm{TeV}\right)$. In the second search, dijet centrality ratio distributions have been measured in the ATLAS detector, with an integrated luminosity of $3.1 \mathrm{pb}^{-1}$. A search for quark compositeness using the model of quark 4-fermion contact interactions has been performed, and such interactions have been excluded at 95\% CL for a compositeness scale below $2.0 \mathrm{TeV}$.
\end{abstract}

Kruger 2010: Workshop on Discovery Physics at the LHC

December 5 - 10, 2010

Kruger National Park, Mpumalanga, South Africa

\footnotetext{
* Speaker.

$\dagger$ On behalf of the ATLAS Collaboration
} 


\section{Introduction}

Two-jet events are described well in the Standard Model (SM) by Quantum Chromodynamics (QCD). However, several extensions beyond the SM predict the existence of new particles with heavy masses, decaying into two energetic jets. Quark compositeness is one such scenario, introducing the quark sub-structure. These sub-structures are visible above a compositeness scale $\Lambda$, below which quarks appear point-like. If this scale is sufficiently low, narrow resonant states of excited quarks could be produced. This can be detected as a difference in shape of the di-jet mass distribution as is shown schematically in Figure 1 (left).
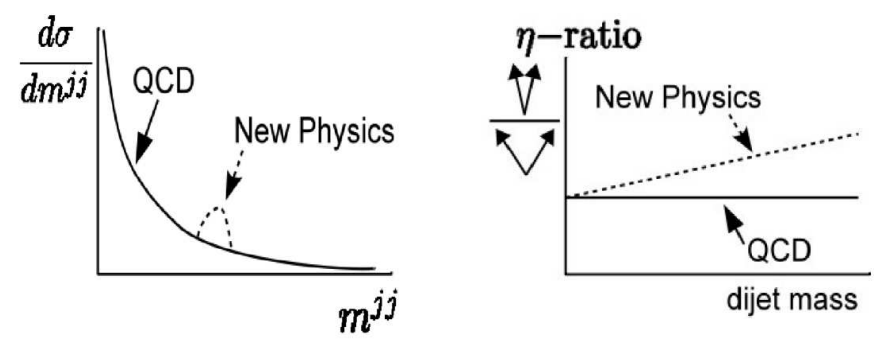

Figure 1: Comparison of QCD dijet mass distribution (left) and centrality ratio (right) (explained in the text) with possible new physics scenarios.

If the scale is much larger than the accessible energy in the colliders, the manifestation of compositeness will be an effective 4-fermion contact interaction. In this case, new processes produce more central activity than QCD, resulting in an increase in the dijet centrality ratio (described in Section 3) in higher dijet masses, as is shown schematically in Figure 1 (right).

In the following sections, these two searches are explained in more details [1-4].

\section{Dijet Resonance Search}

In this search, the dijet invariant mass is the observable, as it is the most sensitive to new physics containing new heavy particles decaying to two jets. It is defined as:

$$
m_{j j}=\sqrt{\left(E_{1}+E_{2}\right)^{2}-\left(\vec{p}_{1}+\vec{p}_{2}\right)^{2}}
$$

The first part of the analysis consists of scanning the dijet mass spectrum to search for any deviations from the smoothly falling distribution of the QCD dijet mass spectrum. In the absence of any deviations, upper limits are set on the cross-section times detector acceptance which, compared to the expected $\mathrm{q}^{*}$ cross-section, yield a lower limit on the mass of the excited quark.

\subsection{Event Selection}

To select dijet events, the leading (i.e. highest $p_{T}$ ) and next-to-leading jets are required to be above 150 and $30 \mathrm{GeV}$ in $p_{T}$ respectively. The cut on the leading jet $p_{T}$ is based on the plateau of the jet trigger used to select events. The cut on the second jet is to ensure high jet reconstruction efficiency. In order to avoid regions where the jet calibration has unknown systematic uncertainties, 
both jets are required to be in the central region: $|\eta|<2.8$, where $\eta$ is the pseudorapidity of each jet. Also, the standard ATLAS-recommended cleaning cuts are applied to the events ${ }^{1}$. An additional cut on the $\eta$-difference of the two leading jets is also applied, based on the difference in event topologies of QCD dijet events, and those coming from the composite quark decay. This cut is based on the optimisation of the Monte Carlo (MC) signal significance: $s / \sqrt{b}$, where $s$ is the MC excited quark signal, and $b$ is the QCD background (Figure 2 right). A value of 1.3 has been chosen for this cut. Also, as the events from new physics tend to be more central, each of the first two leading jets are required to be within $|\eta|$ of 2.5. Finally the dijet invariant mass is required to be $>350 \mathrm{GeV}$ to eliminate any potential kinematic bias from the jet selection requirements.
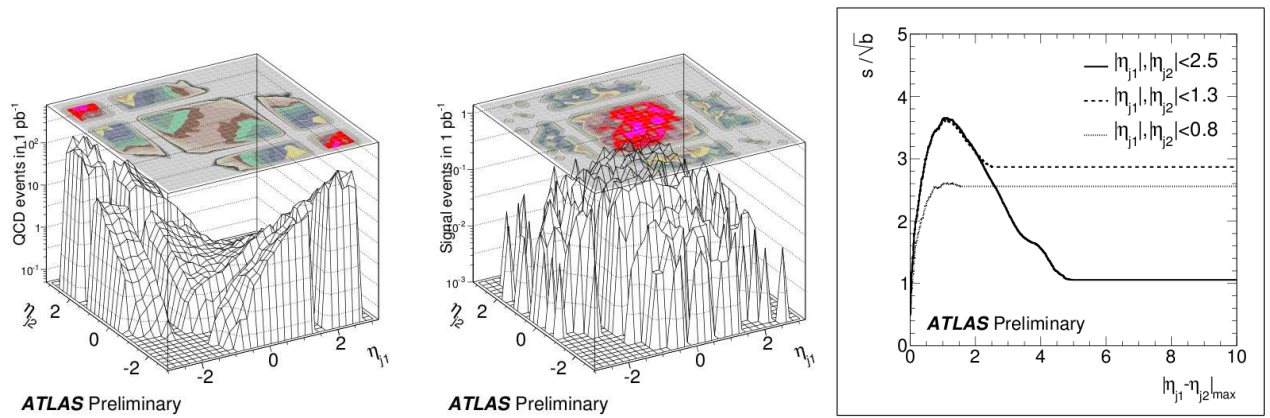

Figure 2: Expected distributions of event yields as a function of pseudo-rapidities of the first two jets in the event; left: QCD events, middle: signal events $\left(m_{q^{*}}=1 \mathrm{TeV}\right)$. Right: $s / \sqrt{b}$ ratio using QCD sample as background and the $1 \mathrm{TeV}$ excited quark sample as signal.

\subsection{Background Determination}

The QCD background is estimated by fitting the following smooth function to the observed data spectrum:

$$
f(x)=p_{1}(1-x)^{p_{2}} x^{p_{3}+p_{4} \ln x}
$$

where $x=m_{j j} / \sqrt{s}$, and $p_{i}, i=1,2,3,4$ are free parameters. It has been shown that this function fits well the QCD mass distribution of PYTHIA, HERWIG, and NLO pQCD predictions for $p \bar{p}$ collisions at $\sqrt{s}=1.96 \mathrm{TeV}$ [6]. Also, MC studies using PYTHIA in ATLAS [7] demonstrate that this function describes the QCD mass spectrum well, with a $\chi^{2} / N D F$ of $27.0 / 22$ over the dijet mass range of $200<m_{j j}<1900 \mathrm{GeV}$, as is shown in Figure 3.

In order to search for a shape difference, various statistical tests that are sensitive to bumpy structures and overall disagreement, have been performed. For all these tests, large p-values of the null hypothesis have been obtained, indicating no significant discrepancy between data and the QCD dijet background. Figure 4 shows the dijet mass spectrum of data, and the fitted function of Equation 2.2; the uncertainties are statistical only.

\subsection{Limits on the mass of the Excited Quark}

As no deviation from the QCD background is observed in data, upper limits have been set on the production cross-section of dijet events, times the detector acceptance. A Bayesian approach

\footnotetext{
${ }^{1}$ Events with poorly measured jets with $p_{T}>15 \mathrm{GeV}$ are vetoed [5].
} 


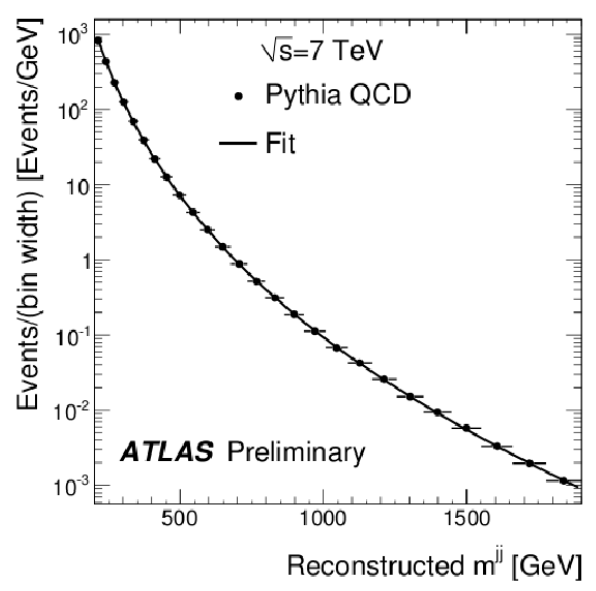

Figure 3: PYTHIA prediction for the dijet mass spectrum, at the leading order.

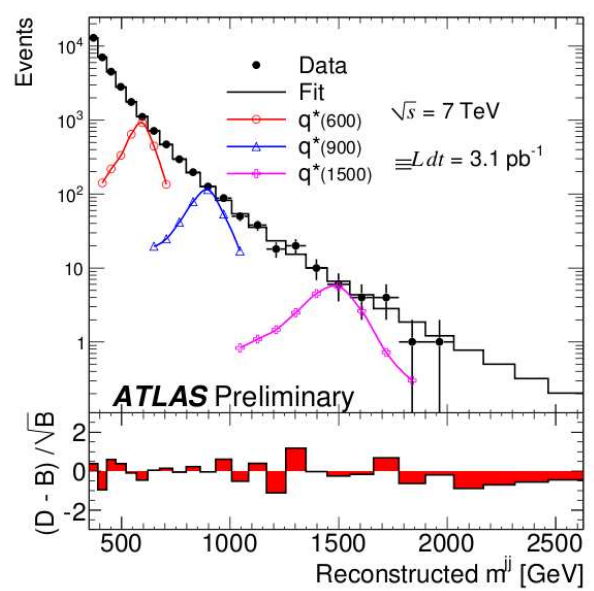

Figure 4: Dijet mass distribution of data, and the fit function of Eq.2.2.

with flat priors in signal yield is used to set the 95\% confidence level limits. In this approach, the likelihood function is a product of Poisson factors computed for each bin of the dijet mass distribution.

Systematic uncertainties are taken into account as nuisance parameters in the calculation of the posterior probability. The main sources of systematic uncertainties are as follows:

- Jet energy scale uncertainty: which is a function of jet $p_{T}$ and $\eta$, and varies between $6 \%-9 \%$.

- Background fit parameters uncertainty: taken from the uncertainty on the parameters resulting from the fit of Eq.2.2 to data.

- Uncertainty on the integrated luminosity: $11 \%$

- Jet energy resolution uncertainty: negligible effect

Figure 5 shows the effect of systematic uncertainties on the posterior distribution for a subset of data, and Figure 6 displays the upper limit on $\sigma . A$ as a function of the resonance mass. 


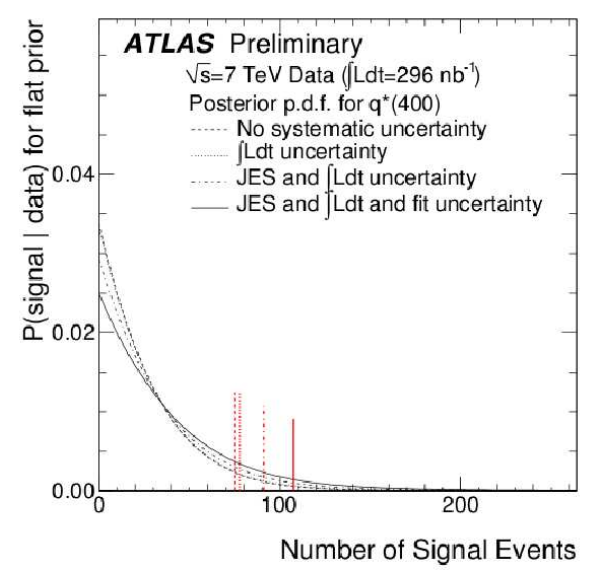

Figure 5: Posterior probability distribution, assuming a flat prior. The Monte Carlo signal used here is an excited quark of mass $400 \mathrm{GeV}$.

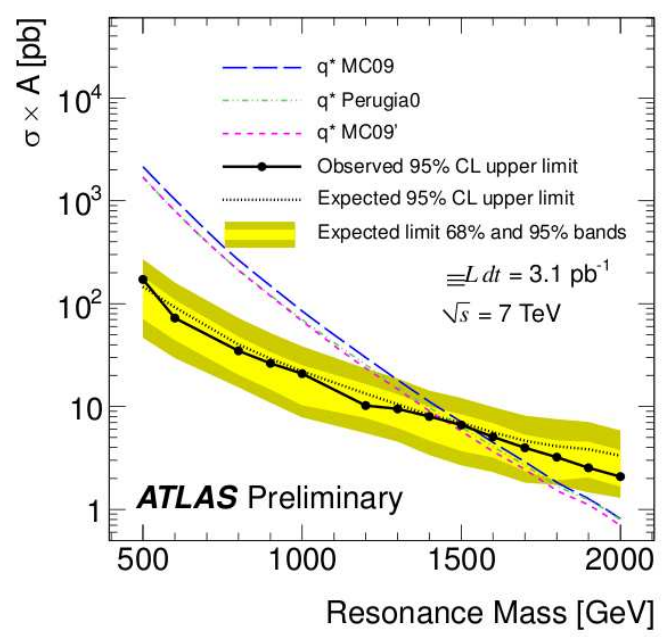

Figure 6: The $95 \%$ CL upper limit on $\sigma . A$ as a function of dijet resonance mass.

A $95 \%$ CL lower exclusion limit of $1.53 \mathrm{TeV}$ is obtained on the excited quark mass by comparing the limit on $\sigma$.A to the theoretical prediction for excited quark production, using MRST2007 PDF set [8].

\section{Dijet Centrality Ratio}

In the second search, the observable is the dijet centrality ratio, defined as:

$$
R_{c}=\frac{N\left(\left|\eta_{1,2}\right|<0.7\right)}{N\left(0.7<\left|\eta_{1,2}\right|<1.3\right)}
$$

where $N$ is the number of events with the first two leading jets in the specified $\eta$ regions.

Since the new physics tend to be more central compared to QCD events (where the t-channel is dominant), a deviation at high dijet masses from the rather flat centrality ratio spectrum of QCD is expected. 


\subsection{Event Selection}

The leading jet $p_{T}$ is required to be above $60 \mathrm{GeV}$, and the second jet $p_{T}$ above $30 \mathrm{GeV}$. The asymmetric thresholds are used in order to allow for a third jet coming from radiation. Each of the two leading jets should be within $|\eta|<1.3$. The event cleaning cuts are also applied. The value of 1.3 for $|\eta|$ is chosen in order to minimise the potential differences in jet response between the central dijet events $(0<|\eta|<0.7)$, and the non-central dijet events $(0.7<|\eta|<1.3)$, since the difference in jet response could have a significant impact on the sensitivity .

\subsection{Comparison to QCD, and setting Limits}

The $\chi^{2}$ goodness-of-fit test is used to compare the centraliy ratio in data with QCD Monte Carlo. Getting a large p-value indicates that there is no significant deviation from the Standard Model QCD prediction.

Two types of systematic uncertainties are considered:

- Experimental uncertainties: jet energy scale uncertainty.

- Theoretical uncertainties: NLO renormalisation and factorisation scales, and Parton Distribution Functions (PDF) uncertainties.

Monte Carlo pseudo-experiments have been generated to convolute these sources of systematic uncertainties.

Figure 7 shows the relative systematic uncertainties with respect to the QCD prediction on the centrality ratio. Figure 8 shows the centrality ratio distribution as a function of the dijet mass, for data and QCD prediction. A Bayesian approach with flat prior in $\frac{1}{\Lambda^{2}}$ is used to set limits on the compositeness scale. This gives a 95\% CL lower limit of $2 \mathrm{TeV}$ on the compositeness scale, $\Lambda$.

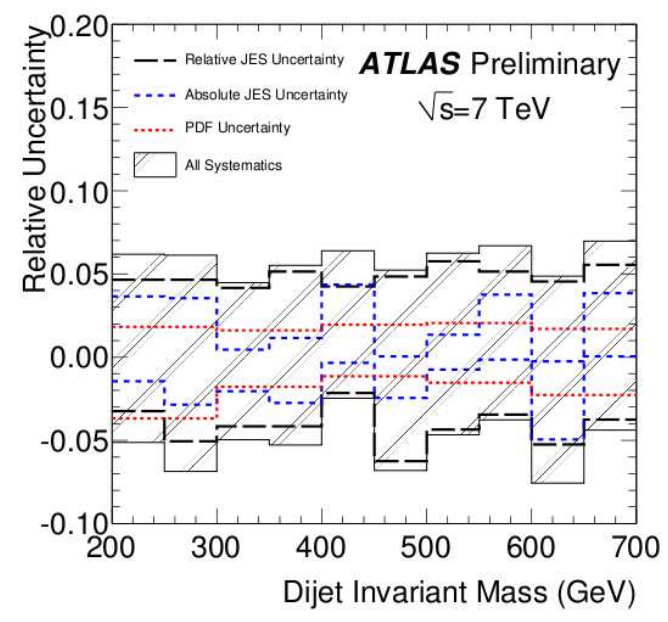

Figure 7: Relative systematic uncertainties of the dijet centrality ratio with respect to QCD prediction. 


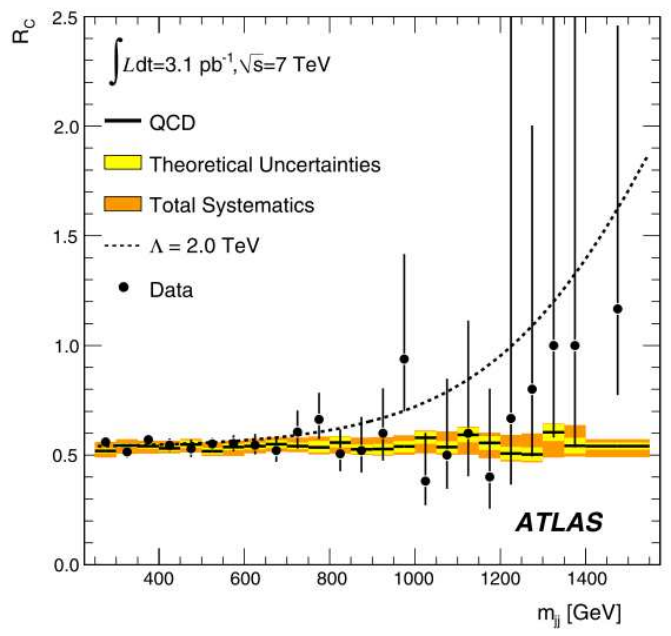

Figure 8: Dijet centrality ratio as a function of dijet mass.

\section{Conclusion}

Two exotic dijet searches have been performed. In both searches data agreed well with the QCD prediction, and no evidence of new physics has been observed. 95\% CL limits have been set on the mass of the excited quark, and on the compositeness scale.

\section{References}

[1] The ATLAS Collaboration, Search for New Particles in Two-Jet Final States in 7 TeV Proton-Proton Collisions with the ATLAS Detector at the LHC, Phys. Rev. Lett 105,16801 (2010) 327-345 [hep-ex/1008-2461v2].

[2] The ATLAS Collaboration, Search for new particles decaying into dijets in proton-proton collisions at sqrts $=7 \mathrm{TeV}$ with the ATLAS detector, ATLAS-CONF-2010-080, CERN, Geneva, Oct, 2010

[3] The ATLAS Collaboration, Search for Quark Contact Interactions in Dijet Angular Distributions in pp Collisions at sqrt(s) = 7 TeV Measured with the ATLAS Detector, Phys. Lett 694 (2011) 327-345 [hep-ex/1009.5069v1].

[4] The ATLAS Collaboration, High-pT dijet angular distributions in pp interactions at $s=7 \mathrm{TeV}$ measured with the ATLAS detector at the LHC, ATLAS-CONF-2010-074, CERN, Geneva, Jul, 2010

[5] The ATLAS Collaboration, Data-Quality Requirements and Event Cleaning for Jets and Missing Transverse Energy Reconstruction with the ATLAS Detector in Proton-Proton Collisions at a Center-of-Mass Energy of $s=7 \mathrm{TeV}$, ATLAS-CONF-2010-038 .

[6] CDF Collaboration, T. Aaltonen et al., Search for new particles decaying into dijets in proton-antiproton collisions at $s=1.96 \mathrm{TeV}$, Phys. Rev.D 79 (2009) 112002.

[7] T. Sjöstrand, S. Mrenna, and P. Z. Skands, PYTHIA 6.4 Physics and Manual, JHEP 05 (2006) 026 [hep-ph/0603175].

[8] A. Sherstnev and R. S. Thorne, Parton Distributions for LO Generators, Eur. Phys J. C55 (2008) 553-575 [hep-ph/0711.2473]. 